\title{
High-dose-rate interstitial brachytherapy for liver metastases: first study from India
}

\author{
Daya Nand Sharma, MD, Sanjay Thulkar, MD, Seema Sharma, Msc, Ajeet Kumar Gandhi, MD, \\ Kunhi Parambath Haresh, MD, Subhash Gupta, MD, Goura Kisor Rath, MD, Pramod Kumar Julka, MD \\ Department of Radiation Oncology. All India Institute of Medical Sciences, New Delhi, India
}

\begin{abstract}
Purpose: To study the safety and efficacy of high-dose-rate interstitial brachytherapy (HDRIBT) in patients with liver metastases (LM).

Material and methods: Between 2009 and 2011, 10 patients with 12 metastatic lesions in the liver were enrolled in this prospective trial. All patients had either refused surgery or found ineligible for surgery due to various factors. Under CT guidance, 16 gauze blind end stainless steel or rigid plastic brachytherapy needle was inserted in the center of lesion through the percutaneous route. Generally, a single interstitial brachytherapy (IBT) needle for lesions up to $3 \mathrm{~cm}$ and multiple needles for lesions more than $3 \mathrm{~cm}$ in diameter were inserted. Treatment was delivered with a single highdose-rate (HDR) dose of 20 Gy prescribed to the target. The needles were removed immediately after the treatment. The endpoints of study were acute complications and local control of the disease.

Results: The median size of the lesion was $3.8 \mathrm{~cm}(2.7-7.0 \mathrm{~cm})$. The average time for the entire IBT procedure was 65 minutes (50-105 minutes). Median follow up was 9 months (3-17 months). None of the patients had fatal complications. Minor complications like pain, nausea/vomiting, and asymptomatic pleural effusion were observed in 3, 2 and 1 patients, respectively. Local control rate at 12 months was $75 \%$. The 1-year local progression free survival (LPFS) was $33 \%$.

Conclusion: Although limited by small sample size, the results of our first study from India suggest that HDRIBT is a safe and effective non surgical option for LM.
\end{abstract}

Key words: liver metastases, interstitial brachytherapy, high-dose-rate.

\section{Purpose}

Liver is one of the organs commonly involved with metastases from various primary malignancies. Surgical resection of LM is the only potentially curative modality, but it is possible in less than 20 percent of patients [1]. Majority of patients are not suitable for surgical resection due to various clinical and technical reasons. For such patients, various non-surgical options are three dimensional conformal radiation therapy (3D-CRT), stereotactic body radiation therapy (SBRT), transarterial chemoembolization (TACE), and various thermo-ablative procedures like radiofrequency ablation (RFA) and laser induced thermal therapy (LITT) [2]. Even for operable lesions, image guided percutaneous ablative procedures are increasingly being used, because they are relatively safer, minimally invasive and equally effective [3]. Interstitial brachytherapy (IBT) is relatively a new and less studied technique of treatment LM [4]. It is preferred over the other thermal ablative techniques for lesions larger than $3-5 \mathrm{~cm}$ in size or located in close proximity to large vessels which are less likely to be ablated due to heat sink effect $[5,6]$. The IBT consists of insertion of single or mul- tiple percutaneous needles in the lesion under ultrasonography (USG) or CT scan guidance, and connecting them to remote after loading brachytherapy machine for delivering a single high-dose-rate (HDR) dose of about $20 \mathrm{~Gy}$. As compared to external beam radiation therapy (EBRT) techniques like 3D-CRT and SBRT, high-dose-rate interstitial brachytherapy (HDRIBT) can potentially provide better dosimetry since tumor movement due to breathing, unlike in EBRT, does not affect dose distribution. Limited experiences through small studies [7-12] exist in the literature regarding the role of HDRIBT in LM and primary hepato-cellular carcinoma, and have shown efficacy and safety. We conducted a prospective trial to study the feasibility of HDRIBT in $\mathrm{LM}$ at our institute. The endpoints of the study were acute complications and local control of the disease.

\section{Material and methods}

From May 2009 to June 2011, 10 patients with 12 metastatic lesions in the liver were enrolled in this prospective trial. All patients had either refused surgery or found inel- 
igible for surgery due to co-morbidities, and had consented for inclusion in the study. Diagnosis of the LM was proved by aspiration cytology or biopsy. Inclusion criteria were as follows: 1) age more than 18 years; 2) Karnofksy Performance Score of 70;3) controlled primary site; 4) liver function tests within normal limits. Exclusion criteria included:1) patients with more than three lesions; 2) pediatric patients; 3) metastases from Wilm's tumor, choriocarcinoma, seminomas or other chemosensitive tumors; 4) patients with metastases to multiple visceral organs; 5) previous irradiation to liver including SBRT. CT imaging was done for all patients as the baseline imaging for determining the disease extent and post treatment response assessment.

\section{Brachytherapy implant procedure}

All the patients were hospitalized one day prior to the procedure. No specific premedication was advised. The procedure was carried out in CT scan room (Phillips Large Bore CT Scanner ${ }^{\circledR}$, Royal Philips Electronics, Amsterdam, The Netherlands) under local anesthesia ( $2 \%$ xylocaine). In the breath hold position, 16 gauze blind end stainless steel or rigid plastic brachytherapy needle was inserted in the center of lesion through the percutaneous route. The needle tip was preferably advanced 3-5 $\mathrm{mm}$ beyond the lesion since the needle tip was blind and could not dwell a source. Caution was taken not to introduce the needle during the breathing movement in order to avoid tissue tear. Numbers of IBT needles were decided by the size and shape of the needle. Usually a single IBT needle for lesions up to $3 \mathrm{~cm}$ and multiple needles for lesions more than $3 \mathrm{~cm}$ in diameter were inserted. The distance between two adjacent needles was kept approximately $2-3 \mathrm{~cm}$. The needles were then secured with screws (Fig. 1). If required, needles were sutured to the skin for additional securing. CT scan images were acquired with a slice thickness of $3 \mathrm{~mm}$ and transferred to treatment planning system.

\section{Brachytherapy planning and treatment delivery}

Brachytherapy planning was performed on PLATO ${ }^{\circledR}$ planning system, version 14.1 (Nucletron, an Elekta com-

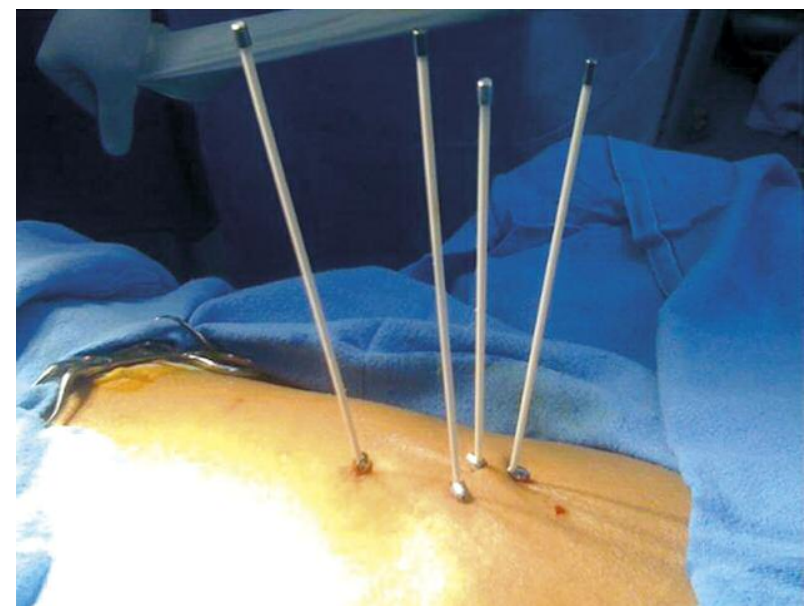

Fig. 1. Clinical photograph of a patient in prone position showing 4 brachytherapy needles fixed with screws pany, Elekta AB, Stockholm, Sweden) using slice thickness of $3 \mathrm{~mm}$. The clinical target volume (CTV) and normal liver volume were delineated on each slice. Implant needles were also marked in order to reconstruct the needle length. No margin was given to CTV to determine planning target volume (PTV). Using step size of $2.5 \mathrm{~mm}$, a plan was generated for a dose of $20 \mathrm{~Gy}$ prescribed to PTV (Fig. 2). Only dwell positions within the PTV were activated. The volume of normal liver (excluding PTV) receiving 5 Gy was kept below $30 \%$. After the plan approval, patient was taken to Microselectron $\mathrm{HDR}^{\circledR}$ brachytherapy unit (Nucletron, an Elekta company, Elekta AB, Stockholm, Sweden) using Ir-192 radioactive source with initial activity of $10 \mathrm{Ci}$ for treatment delivery. Treatment was delivered in a single fraction of 20 Gy. The needle was removed in the breath hold position immediately after the completion of treatment and puncture site was sealed. The patients were kept in the indoor unit overnight for observation.

\section{Assessment of complications}

Acute complications were defined as those occurring within 6 months of the treatment. This included complications encountered during and within one week of the procedure (perioperative complications). Major complications were defined as those resulting in life threatening condition (like intra-abdominal hemorrhage) requiring emergency therapies like blood transfusion and endotracheal intubation. Minor complications included the nausea/vomiting, pain requiring analgesics, minor bleeding which did not require any active or resuscitative measures.

\section{Follow up and response assessment}

Patients were followed up every month till a period of 6 months and then every 3 months. CT scan was done every 3 months to assess status of the treated lesion. Response evaluation was done using the criteria [13] defined by Response Evaluation Criteria In Solid Tumors (RECIST). First assessment CT scan was performed at 3 months post procedure in view of the post treatment edema and slow regression following large single dose of $20 \mathrm{~Gy}$. Local control was de-

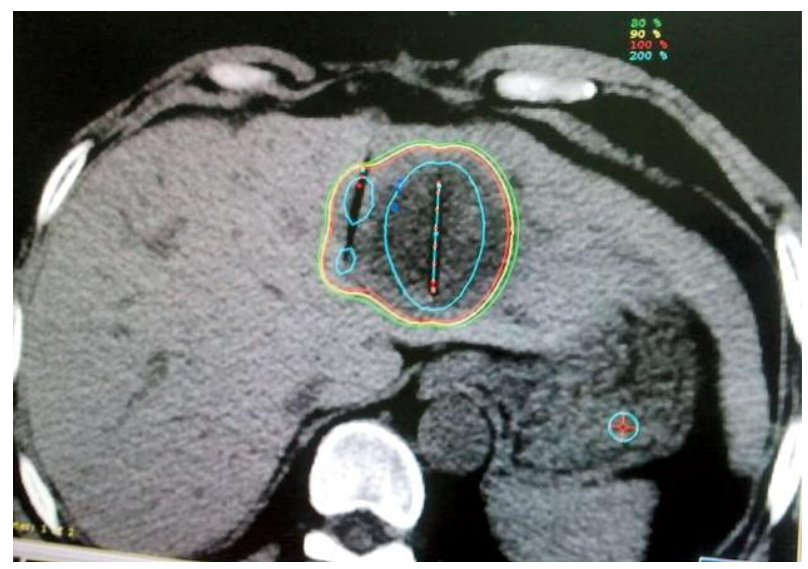

Fig. 2. Planning CT scan of a patient showing the metastatic lesion, two brachytherapy needles and dosimetry. The red color line represents the prescription isodose line (20 Gy) and cyan color line represents the $200 \%$ isodose line ( $40 \mathrm{~Gy}$ ) which covers a significant central part of the lesion 
Table 1. Patient characteristics

\begin{tabular}{lc}
\hline Gender & No. of patients \\
\hline Male & 5 \\
\hline Female & 5 \\
\hline Median & Years \\
\hline Range & 54 \\
\hline Primary site & $40-72$ \\
\hline Breast & No. of patients \\
\hline Colorectal & 3 \\
\hline Gall Bladder & 2 \\
\hline Stomach & 2 \\
\hline Unknown origin & 2 \\
\hline Number of lesions & 1 \\
\hline Solitary & No. of patients \\
\hline Two & 8 \\
\hline Size of the lesion & 2 \\
\hline Median & cm \\
\hline Range & 3.8 \\
\hline Previous treatment & $2.7-7.0$ \\
\hline RFA & No. of patients \\
\hline Surgery & 2 \\
\hline None & 1 \\
\hline Disease free interval & 7 \\
\hline Median & Months \\
\hline Range & 14 \\
\hline - centimeter, RFA - radiofrequency ablation & \\
\hline
\end{tabular}

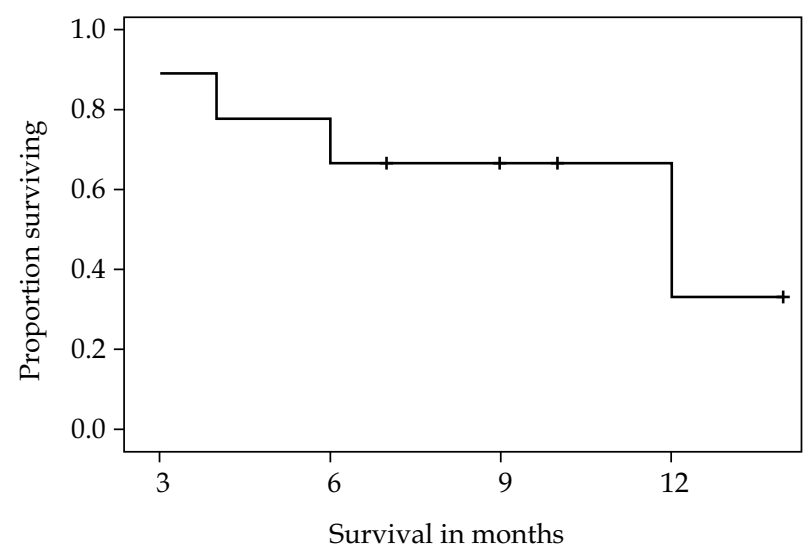

Fig. 3. The Kaplan Meier curve showing local progression free survival

fined as absence of more than $20 \%$ increase in the size of treated lesion compared with baseline. Appearance of any new lesion outside the treated area was not taken as local recurrence. Local progression free survival (LPFS) was defined as the period from date of enrollment until the date of local disease progression (more than $20 \%$ increase in the
Table 2. Details of brachytherapy dosimetry

\begin{tabular}{|c|c|}
\hline Duration of brachytherapy procedure & Minutes \\
\hline Median & 65 \\
\hline Range & $50-105$ \\
\hline No. of brachytherapy needles & $N$ \\
\hline Median & 2 \\
\hline Range & $1-4$ \\
\hline Target volume & c.c. \\
\hline Median & 21.3 \\
\hline Range & $9-84$ \\
\hline $\mathrm{V}_{100}$ & c.c. \\
\hline Median & 19.6 \\
\hline Range & $9.3-83.2$ \\
\hline$V_{150}$ & c.c. \\
\hline Median & 10.2 \\
\hline Range & 5.1-28.4 \\
\hline$V_{200}$ & c.c. \\
\hline Median & 6.9 \\
\hline Range & $3.7-16.2$ \\
\hline Liver volume receiving > 5 Gy & $\%$ \\
\hline Median & 14 \\
\hline Range & $4-19$ \\
\hline \multicolumn{2}{|c|}{$\begin{array}{l}\text { C.c. - cubic centimeter, } V_{100} \text {-volume receiving } 100 \% \text { of prescription dose, } \\
V_{150} \text {-volume receiving } 150 \% \text { of prescription dose, } V_{200} \text {-volume receiving } \\
200 \% \text { of prescription dose }\end{array}$} \\
\hline
\end{tabular}

size of treated lesion), or death, or last follow up whichever came first. The survival was calculated by Kaplan Meier survival method [14]. Statistical analysis was performed using the statistical software SPSS, version 17.

\section{Results}

A total of 12 lesions were treated in 10 patients. Clinical characteristics of the patients are presented in Table 1 . There were 5 males and 5 females with a median age 54 years (range 40-72). The median size of the lesion was $3.8 \mathrm{~cm}$ (range 2.7-7.0 cm). Single needle was used in 5 patients while multiple needles were used in 5 patients. In one patient, maximum of 4 needles were used for lesion size of $7 \mathrm{~cm}$ (Fig. 1). The average time for the entire IBT procedure (needle insertion to needle removal) was 65 minutes (range of 50-105 minutes). All patients could complete the prescribed treatment dose of $20 \mathrm{~Gy}$. The details of brachytherapy dosimetry are shown in Table 2 . The volume of normal liver (excluding the target volume) receiving more than 5 Gy did not exceed $19 \%$ in any of the patient. Table 3 shows the various major and minor complications. None of the patients had fatal complications. Minor complications like pain, nausea, vomiting were well managed with simple drugs. Pain was the most frequent minor complication encountered in 3 patients. One patient who had minimal pleural effusion without any symptom was managed with observation alone and no active intervention was required. All the patients 
Table 3. Number of patients showing major and minor complications ( $n=10$ patients)

\begin{tabular}{|c|c|c|c|}
\hline & Complication & No. of patients & Intervention \\
\hline \multirow[t]{2}{*}{ Complications during procedure } & Abdominal hemorrhage (major) & 0 & - \\
\hline & Anaphylaxis/shock due to local anesthetic (major) & 0 & - \\
\hline \multirow{5}{*}{$\begin{array}{l}\text { Complications during } \\
\text { perioperative period }\end{array}$} & Pain (minor) & 3 & Simple oral analgesics \\
\hline & Nausea/vomiting (minor) & 2 & Antiemetic drugs \\
\hline & Asymptomatic pleural effusion (minor) & 1 & No active treatment \\
\hline & Abdominal hemorrhage (major) & 0 & - \\
\hline & Jaundice (major) & 0 & - \\
\hline
\end{tabular}

were discharged from the hospital within 24 hours of the procedure. Median follow up duration was 9 months (3-17 months). At 12 months, 3 out of 12 lesions showed local progression and thus local control rate was $75 \%$. All these 3 patients developed metastases to other visceral organs as well (lung 2, brain 1). Of the 7 patients (with 9 lesions under control), 2 patients developed metastases to bones, lung and liver. The 1-year LPFS was 33\% (Fig. 3).

\section{Discussion}

Among non surgical options for patients with LM, RFA is relatively more popular method of ablation. However, it has limitations concerning the number, localization, as well as size and shape of the LM, with the risk of complications ranging from $8-35 \%[15,16]$. CT guided HDRIBT is a new treatment for LM with only limited studies [7-12] in the literature reporting local control rates of $87-93 \%$; similar to other ablative therapies [2]. Unlike RFA, HDRIBT is not affected by nearby blood vessel or size of the lesion. Compared to EBRT treatment methods like SBRT, HDRIBT dosimetry remains unaffected by respiratory movements, because the implanted needle moves with target. Therefore, it precisely delivers a large single dose of radiation to the target lesion and minimal dose to the surrounding liver parenchyma which has limited radiation tolerance. This makes it a highly conformal treatment with good therapeutic potential, while experience is limited. To the best of our knowledge, ours is the first such study being reported from India so far, and our results have shown that HDRIBT is a safe and feasible non surgical option for LM in our setup.

The largest experience in the literature has been reported by Ricke et al. [7-9]. In their first study, [7] 19 patients of LM were treated by HDRIBT dose of $17 \mathrm{~Gy}$ (range 12-25 Gy). They observed local control rate of $53-71 \%$ at 1 year. Two patients (10\%) experienced severe side effects, one had obstructive jaundice due to irradiation edema, and the other had intra-abdominal hemorrhage. In their second study, Ricke et al. [8] treated 35 patients with mean tumor size of $4.6 \mathrm{~cm}$ (range, $2.5-11 \mathrm{~cm}$ ) with either HDRIBT alone using median dose of $17 \mathrm{~Gy}$ (range, 10-20 Gy) or in combination with thermal ablation. Severe complications were recorded in $2(5 \%)$ patients. The local control rate after 6 months was $87 \%$ and $73 \%$ for IBT alone and combined treatment, respectively. In their third study [9], they used three different dose levels (15, 20 and $25 \mathrm{~Gy})$, and observed a statistically significant better local control with $25 \mathrm{~Gy}$ as compared to 20 Gy or 15 Gy.

Table 4 summaries the results of various studies [7-12] published so far relating to use of HDRIBT in LM. The results of our study may not be strictly compared with others in the literature due to small sample size and short follow up. Although local control rates in our study were slightly inferior to other studies, possibly due to small sample size and heterogeneous population of patients having different primary sites; but our severe complication rate of $0 \%$ has been least of all the studies. The 1-year LPFS in our study is consistent with other studies by Ricke et al. [8] and Ricke et al. [7] (33\% vs. 34\% vs. 33\%).

Table 4. Studies published regarding the use of HDRIBT for liver metastases

\begin{tabular}{|c|c|c|c|c|c|c|c|c|c|c|}
\hline Author & $\begin{array}{l}\text { No. of } \\
\text { pts }\end{array}$ & $\begin{array}{l}\text { No. of } \\
\text { pts with } \\
\text { LM }\end{array}$ & $\begin{array}{l}\text { Primary } \\
\text { site }\end{array}$ & $\begin{array}{c}\text { Median } \\
\text { lesion } \\
\text { size }(\mathrm{cm})\end{array}$ & $\begin{array}{c}\text { No. of } \\
\text { catheter } \\
\text { (median) }\end{array}$ & $\begin{array}{l}\text { Dose } \\
\text { (Gy) }\end{array}$ & $\begin{array}{c}\text { Median } \\
\text { FU } \\
\text { (months) }\end{array}$ & $\begin{array}{l}\text { Local } \\
\text { control } \\
(\%)\end{array}$ & Survival & $\begin{array}{c}\text { Major } \\
\text { complica- } \\
\text { tion } \\
\text { rate }(\%)\end{array}$ \\
\hline Ricke et al. 2004 [7] & 20 & 19 & Mixed & 5 & $2-6$ & 17 & 13 & $53-71$ & 1-yr PFS, 33\% & 10 \\
\hline Ricke et al. 2004 [8] & 37 & 35 & Mixed & 4.6 & NR & 17 & 14 & 73 & 1-yr PFS, 34\% & 5 \\
\hline Ricke et al. 2010 [9] & 73 & 73 & Colo-rectum & 3.1 & NR & $\begin{array}{l}15 \\
20 \\
25\end{array}$ & 15 & 75 & $N R$ & 7.5 \\
\hline Steffen et al. 2010 [10] & 19 & 19 & Colo-rectum & NR & $2-6$ & 20 & 9 & 60 & NR & NR \\
\hline Wieners et al. 2011 [11] & 41 & 41 & Breast & 4.6 & NR & 18.5 & 18 & 93 & 1-yr PFS, $40 \%$ & 1.5 \\
\hline Tselis et al. 2012 [12] & 31 & 23 & Mixed & NR & 3 & 13 & 13 & 79 & 1 -yr OS, $66 \%$ & 4.7 \\
\hline
\end{tabular}

LM - liver metastasis, Pts - patients, IBT- interstitial brachytherapy, LC- local control, PFS- progression free survival, HDR- high-dose-rate; FU-follow up 
Although most studies have used large single dose of HDR, but recently, Tselis et al. [12] reported their results of 31 patients using a fractionated schedule of HDRIBT (twice a day) with total dose ranging from 7-32 Gy. The dose per fraction varied from 4-14 Gy. In their series, 23 patients had LM from different primary sites. They observed local control rates of $79 \%$ at 1 year and $59 \%$ at 2 year with $4.7 \%$ severe complication rate. Most series including ours (Table 4) have included patients with heterogeneous primary sites and therefore results vary across these studies. However, two studies by Ricke et al. [9] and Steffen et al. [10] have included patients with LM from colorectum only, and one study by Wieners et al. [11] had primary site in the breast alone. The best local control rates $(93 \%)$ have been observed in series having breast primary, but the 1-year PFS of $40 \%$ is apparently same compared to rest of the studies. In terms of toxicity, HDRIBT seems to be safe as the procedure or radiation related severe complications rate has been found to be within acceptable limits $(1.5-10 \%)$ in the published literature so far. Several possible factors namely target volume, irradiation time, number of needle/catheters has been theoretically associated with toxicity, but none has been proved in the literature so far. Minor complications like pain, nausea, vomiting, and asymptomatic pleural effusion etc. occurring 12 to 24 hours after treatment have been reported in about $10 \%$ of patients [4]. Major complications, though rare, include liver abscess and severe abdominal bleeding. We did not observe any major complication in our study.

SBRT is a contemporary radiation treatment options for LM and comparison with HDRIBT inescapable. SBRT re-fers to the delivery of large doses of highly conformal radiation with steep dose gradients toward the surrounding normal tissue over a limited number of fractions ( 1 to 6 fractions) to extra cranial tumor sites [17]. It is a simple, convenient and non-invasive method of treatment, and therefore rapidly becoming popular. Though metastatic lesions up to $6 \mathrm{~cm}$ in liver have been treated, but lesion size between $3-5 \mathrm{~cm}$ is the ideal one. Unlike HDRIBT, lesions located in close proximity to bowel lumen or chest wall/ribs are avoided. Although uncertainty related to breathing movements is minimal with the use of immobilization devices, abdominal compression and gating techniques; the dosimetric variation cannot be ruled out during treatment delivery. Shift in liver position relative to the vertebral bodies can be as large as $1 \mathrm{~cm}$ from fraction to fraction [18]. In HDRIBT, breathing motion is not a limiting factor since the catheter has a constant position relative to the target without further specific preparations. Due to sharp dose gradient within and outside the target, normal liver receives minimal doses and the center of the tumor receives 2-3 times higher dose than the prescription dose (Fig. 2). Although there are no studies comparing SBRT and HDRIBT in LM, local control rates seem to be comparable. Severe toxicity related to SBRT is uncommon, but likely to be more in patients receiving higher doses to bowel or large volumes of liver. Major complications in the form of hemorrhagic gastritis, rib fracture or chest wall necrosis have been rarely observed [19]. As per the Paris system [20] of dosimetry, multiple needles should be inserted for adequate coverage of lesions larger than $1.5 \mathrm{~cm}$ in diameter. We used single needle for lesions up to
$3 \mathrm{~cm}$ in diameter and accepted the inhomogeneous dosimetry in the tumor. Such inhomogeneity, which is not usually acceptable in routine HDRIBT procedures like breast and prostate brachytherapy, actually proves advantageous in liver tumors. Firstly, the central hypoxic area of the tumor, which is closure to needle, definitely receives much higher dose (>50 Gy for) as compared to the peripheral portion (20 Gy) [21], and therefore may result in higher tumor control. Secondly, multiple needle insertion will definitely enhance the risk of trauma and associated complications.

We realize the limitations of our study: 1) small sample size and 2) short follow up duration. Yet, our results consolidate the safety aspect of HDRIBT in patients of LM and should encourage the readers to further study this topic.

\section{Conclusions}

Ours is the first study being reported from India so far on the use of HDRIBT in patients with LM. Although limited by small sample size, the results of our study suggest that HDRIBT is a safe and effective non surgical option for LM in our setup. Addition of our results to the existing literature would consolidate the practice of HDRIBT, a technique with excellent therapeutic potential, but relatively under-utilized in LM, and encourage us and the readers to design future multi-centric randomized controlled trials with larger sample size and longer follow up.

\section{References}

1. Bentrem DJ, Dematteo RP, Blumgart LH. Surgical therapy for the metastatic disease to the liver. Ann Rev Med 2005; 56: 139-156.

2. Dick EA, Taylor-Robinson SD, Thomas HC et al. Ablative therapy for liver tumors. Gut 2002; 50: 733-739.

3. Fong Y, Salo J. Surgical therapy of hepatic colorectal metastasis. Semin Oncol 1999; 26: 514-523.

4. Ricke J, Wust P. Computed tomography-guided brachytherapy for liver cancer. Semin Radiat Oncol 2011; 21: 287-293.

5. Galandi D, Antes G. Radiofrequency thermal ablation versus other interventions for hepatocellular carcinoma. Cochrane Database Syst Rev 2002; (3): CD003046.

6. Curley SA, Izzo F. Radiofrequency ablation of primary and metastatic hepatic malignancies. Int J Clin Oncol 2002; 7: 72-81.

7. Ricke J, Wust P, Wieners G et al. Liver malignancies: CT-guided interstitial brachytherapy in patients with unfavorable lesions for thermal ablation. J Vasc Interv Radiol 2004; 15: 12791286.

8. Ricke J, Wust P, Stohlmann A et al. CT-guided interstitial brachytherapy of liver malignancies alone or in combination with thermal ablation: Phase I/II results of a novel technique. Int J Radiat Oncol Biol Phys 2004; 58: 1496-1505.

9. Ricke J, Mohnike K, Pech M et al. Local response and impact on survival after local ablation of liver metastases from colorectal carcinoma by computed tomography-guided highdose-rate brachytherapy. Int J Radiat Oncol Biol Phys 2010; 78: 479-485.

10. Steffen IG, Wust P, Rühl R et al. Value of combined PET-CT for radiation planning in CT-guided percutaneous interstitial high-dose-rate single-fraction brachytherapy for colorectal liver metastases. Int J Radiat Oncol Biol Phys 2010; 77: 1178-1185.

11. Wieners G, Mohnike K, Peters N et al. Treatment of hepatic metastases of breast cancer with CT-guided interstitial brachytherapy - a phase II-study. Radiother Oncol 2011; 100: 314-319. 
12. Tselis N, Chatzikonstantinou G, Kolotas C et al. Hypofractionated accelerated computed tomography-guided interstitial high-dose-rate brachytherapy for liver malignancies. Brachytherapy 2012; 11: 507-514.

13. Therasse P, Arbuck SG, Eisenhauer EA et al. New guidelines to evaluate the response to treatment in solid tumors. J Natl Cancer Inst 2000; 92: 205-216.

14. Kaplan EL, Meier P. Nonparametric estimation from incomplete observations. J Am Stat Assoc 1958; 53: 457-481.

15. Liapi E, Geschwind JF. Transcatheter and ablative therapeutic approaches for solid malignancies. J Clin Oncol 2007; 25: 978-986.

16. Tateishi R, Shiina S, Teratani $T$ et al. Percutaneous radiofrequency ablation for hepatocellular carcinoma: an analysis of 1000 cases. Cancer 2005; 103: 1201-1209.

17. Potters L, Kavanagh B, Galvin JM et al. American Society for Therapeutic Radiology and Oncology (ASTRO) and American College of Radiology (ACR) practice guideline for the performance of stereotactic body radiation therapy. Int J Radiat Oncol Biol Phys 2010; 76: 326-332.

18. Case RB, Sonke JJ, Moseley DJ et al. Inter- and intrafraction variability in liver position in non-breath-hold stereotactic body radiotherapy. Int J Radiat Oncol Biol Phys 2009; 75: 302-308.

19. Høyer M, Swaminath A, Bydder S et al. Radiotherapy for liver metastases: a review of evidence. Int J Radiat Oncol Biol Phys 2012; 82: 1047-1057.

20. Pierguin B, Dutreix A, Paine $C$ et al. The Paris system in interstitial radiation therapy. Acta Radiol Oncol 1978; 17: 33-48.

21. Paul JM, Koch RF, Philip PC. Uniform analysis of dose distribution in interstitial brachytherapy dosimetry systems. Radiother Oncol 1988; 13: 105-125. 